\title{
Modeling and Analysis of a Large-Scale Double-Level Guyed Mast for Membrane Antennas
}

\author{
Bingyan Li, ${ }^{1}$ Yuxuan Liu, ${ }^{2}$ Rongqiang Liu, ${ }^{1}$ Hongwei Guo $\mathbb{D}^{1},{ }^{1}$ Qiang Cong, ${ }^{3}$ Qiuhong Lin, ${ }^{3}$ \\ and Shangling Qiao ${ }^{1}$
}

\author{
${ }^{1}$ State Key Laboratory of Robotics and System, Harbin Institute of Technology, Harbin 150001, China \\ ${ }^{2}$ School of Mechanical Engineering and Automation, Beihang University, Beijing 100191, China \\ ${ }^{3}$ China Academy of Space Technology, Beijing 100094, China
}

Correspondence should be addressed to Hongwei Guo; guohw@hit.edu.cn

Received 10 August 2020; Revised 14 October 2020; Accepted 5 November 2020; Published 25 November 2020

Academic Editor: Ali Ramazani

Copyright (c) 2020 Bingyan Li et al. This is an open access article distributed under the Creative Commons Attribution License, which permits unrestricted use, distribution, and reproduction in any medium, provided the original work is properly cited.

\begin{abstract}
This paper proposes a double-level guyed membrane antenna for stiffness improvement of a large-scale tri-prism deployable mast using the collapsible tubular mast (CTM). Initially, the construction of the antenna and the modeling of the CTM boom are illustrated. Afterwards, the central mast with isosceles triangular cross section is mathematically equivalent to a continuum beam, in which the equations of motion and the constitutive relations are derived. Based on the equivalent central beam, the double-level guyed mast for the membrane antenna is modeled as a 2(3-SPS-S) mechanism, and then velocity Jacobian matrices and stiffness matrices of SPS branches are constructed. Additionally, the total stiffness matrix of the equivalent mechanism is derived with the principle of virtual work and then evaluated as an accurate approach. Finally, with the aim to improve the static stiffness of the double-level guyed mast, the numerical analysis using the Genetic Algorithm (GA) is carried out for optimizing the distribution of guys in terms of anchor positions and attachment heights.
\end{abstract}

\section{Introduction}

The increasing multiplicity and complexity of spacecrafts generates the need for large-scale and lightweight antennas. As a thin foldable material, the membrane is particularly suitable for space missions and commonly used to construct antenna reflectors. The application potential of a membrane antenna is expected to be fulfilled by a stable and deployable support structure. To address this issue, the Institute of Structural Mechanics of the German Aerospace Center developed the ultra-lightweight deployable boom and proved its applicability using a $20 \mathrm{~m} \times 20 \mathrm{~m}$ demonstrator [1]. Since then, several deployable composite boom concepts have been developed, such as the sheath-based rollable lenticular-shaped and low-friction (SHEARLESS) boom, the triangular rollable and collapsible (TRAC) boom, X boom, and the collapsible tubular mast (CTM) boom [2]. The analysis object in this research is constructed with the CTM boom, which is the most widely used deployable boom. The characteristics of the CTM boom have been investigated numerically and experimentally. Bai et al. analyzed the geometrical and mechanical properties of the CTM boom in fold deformation and assessed the in-plane strain and interlaminar shear stress on the bonding interface [3]. Chu and Lei studied the mechanical design, dynamic analysis, and mechanical behavior of the CTM boom [4]. Firth and Pankow exerted buckling tests on the CTM boom supported by a conformal root and found that the bending and torsion are linear with the load before buckling point [5]. Hu et al. showed the linear displacement-load curve before local buckling in the axial direction of the CTM boom through axial compression experiments [6].

Two parallel CTM booms are able to support the membrane in an antenna, but the stiffness is hard to meet the standard for large-scaled antennas. Therefore, three parallel CTM booms are used to construct a CTM truss which has a triangular cross section, thus improving the stiffness of the membrane antenna. Murphey proposed methods for 
weight-optimal design of booms and for the rational judgment of boom performance [7, 8]. Eiden et al. presented a continuous-longeron space mast which can change from a helical coiled state into a straight deployed configuration [9]. However, for the large-scaled membrane antenna, the space thermal environment will inevitably induce deformation to the supporting structure, affecting the shape accuracy of the membrane [10]. Therefore, it is crucial to reduce the deformation by improving the static stiffness. For this purpose, it is in need to find a commonly accepted methodology to design the guyed mast which consists of a tall mast laterally supported at several levels along its height by sets of inclined pretensioned guys spaced around the mast [11]. Xiao et al. employed four tension cables to improve the stiffness of a membrane antenna supported by two compliant booms and established an equivalent 4-SPS-S parallel mechanism of the membrane antenna frame [12]. Páez and Sensale modeled the mast as a continuous equivalent beam-column on nonlinear elastic supports in guyed masts, which used the stability functions based on the Timoshenko beam-column [13]. The nonlinear behaviors of guyed masts have been widely studied. Belevičius et al. simplified the nonlinear behavior of the guyed mast by idealizing the nonlinear guys as approximate boundary conditions to optimize the mast structure for self-weight and wind loading [14]. Heydari et al. divided the guyed mast into linear and nonlinear parts and analyzed them separately and optimized the sizes of members, initial cable tensions, the positions of anchor on the ground, and tie level of cables on the mast [15]. Ballaben and Rosales established a nonlinear 3D finite element formulation to study the dynamic properties and the dynamic response of a 3D guyed mast [16]. Besides, Alshurafa et al. developed several nonlinear finite element models to explore the effects of parameters on the strength and stiffness of the guyed masts [17]. Some optimizations and parametric analyses have been carried out as well. Belevičius et al. optimized the double-level guyed mast to obtain the minimum weight design using the Genetic Algorithm and simulated annealing algorithm $[18,19]$ and revealed the dependence of the mast mass on the number of guys' levels [20]. Moreover, Elena Parnás et al. discussed the influence of asymmetrical anchors on behavior of the guyed mast under wind loads [21].

To reduce the computational effort in the pre-design stages, a mast is usually modeled as a continuous equivalent beam-column. The continuum modeling offers a practical and efficient approach for the analysis and design of largescaled structures, thus having a wide range of applications. Considering local effects in each repeating mast element, Noor et al. developed continuum models for large beamlike lattice trusses subjected to static, thermal, and dynamic loadings [22, 23]. Salehian et al. derived the governing partial differential equations of vibration of a repeated-type structure into a Timoshenko equation [24]. Zhang et al. evaluated the equivalent bending stiffness of a hybrid decktruss structure using the equivalent continuum beam method based on the homogenization concept and shearing equivalence principle [25]. In addition, Liu et al. established an equivalent dynamic model for the prestressed space antenna truss with the static condensation method on the bases of the energy equivalence principle and Timoshenko beam theory [26].

For stiffness analysis, the guyed mast is more complex than the mast without guys to be analyzed. The analysis of the guyed mast used to mainly depend on the nonlinear finite element method, which takes much computational effort. Therefore, it is necessary to establish the equivalent mechanism of the guyed mast. Xiao et al. employed four tension cables to improve the stiffness of a membrane antenna in which the one-guy-level mast is modeled as a 4-SPS$S$ parallel mechanism [12]. Dai and Ding established the equilibrium between the twist deflection, the supporting wrenches, and the external wrench on a three-legged compliant platform and for the first time developed an analogy between the feeder and the parallel mechanisms [27]. Based on the principle of virtue work and the determined forces, $\mathrm{Lu}$ and $\mathrm{Hu}$ proposed an equivalent mechanism approach for establishing the stiffness matrices of parallel kinematic machines to solve the elastic deformation [28].

This paper proposes a new modeling and analysis methodology for guyed masts, especially for double-level guyed masts with isosceles triangular cross section. Initially, the design of the membrane antenna and the characteristics of the CTM boom are illustrated. Afterwards, the central mast is mathematically modeled as a continuous equivalent beam in consideration of the material and geometric parameters of each element. Then, the double-level guyed mast is modeled as a 2(3-SPS-S) mechanism, and the total stiffness matrix of the equivalent mechanism is derived. Finally, the influence of anchor positions and attachment heights of guys on the stiffness performance is revealed, and the optimal distribution of guys is proposed based on the Genetic Algorithm (GA).

\section{Structural Characteristics of Membrane Antenna}

In case of lateral deformation, a double-level guyed mast shown in Figure 1 is proposed to strengthen the structural static stiffness and improve the shape accuracy of the membrane. With one additional level of guys to the traditional single-level guyed mast, the constraints for the mast are increased, thus enhancing the static stiffness. In the membrane antenna, a large-scaled strip membrane is attached to the deployable central mast which has an isosceles triangular cross section. There are two lateral battens of equal length and one bottom batten in the section frame. The cross section of each batten is tube-shaped, and the outer diameter and thickness of each batten tube are denoted as $d_{b}$ and $t_{b}$, respectively. Three conformal connections fixed onto the three CTM booms in the deployment process are located at each vertex connecting neighbor battens. Three battens and three conformal connections construct an integrated frame to support the CTM booms. The width and height of the triangular frame are denoted as $b_{0}$ and $h_{0}$, respectively. Three deployment mechanisms forming an isosceles triangle are fixed on the base, and each of them can deploy a CTM 


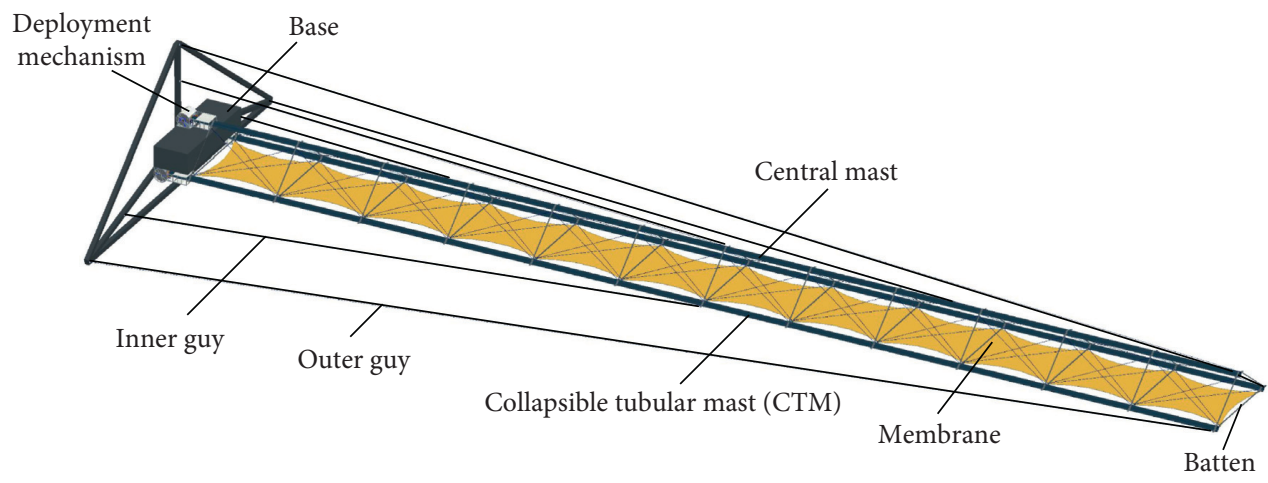

Figure 1: Double-level guyed mast for the membrane antenna.

boom from the base outwards. A continuous CTM boom is divided into a number of longerons by the frames, and the central mast is divided into several repeating units as well. There are two diagonal cables distributed on each rectangular face of the mast unit. When the central mast is fully deployed, it is supported by two levels of guy cables. Each of the three outer guys is anchored on the base and attached to the corresponding vertex of the outermost triangular frame. Meanwhile, each of the three inner guys is anchored on the base as well and attached to the corresponding vertex of a triangular frame between the base and the outermost frame.

As shown in Figure 2, the cross section of a CTM boom is symmetric, and a quarter of its inner profile is illustrated by line $A^{\prime} B^{\prime} C^{\prime} D^{\prime}$. The thickness of the CTM boom is denoted as $t$. The radii of arcs $A^{\prime} B^{\prime}$ and $B^{\prime} C^{\prime}$ are both denoted as $R_{l}$. Straight line $C^{\prime} D^{\prime}$ indicates the bonding plane of the top and bottom parts and has a width of $q$.

The $x^{\prime}$-direction of a CTM boom is along its axis and the cross section is on the $y^{\prime} z^{\prime}$-coordinate plane, which is shown

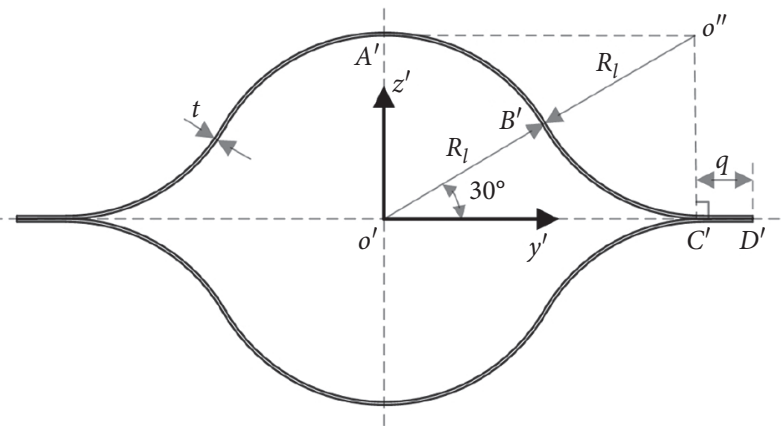

Figure 2: Cross section of the CTM boom.

in Figure 2. The moment of area about the $y^{\prime}$-axis, denoted as $I_{y_{1}}$, and that about the $z^{\prime}$-axis, denoted as $I_{z_{1}}$, are, respectively, expressed as

$$
\begin{aligned}
& I_{y_{1}}=4 \int_{0}^{(\sqrt{3} / 2) R_{l}} t R_{l} \sqrt{R_{l}^{2}-y^{2}} \mathrm{~d} y+4 \int_{(\sqrt{3} / 2) R_{l}}^{\sqrt{3} R_{l}} t R_{l} \frac{\left(R_{l}-\sqrt{R_{l}^{2}-\left(y-\sqrt{3} R_{l}\right)^{2}}\right)^{2}}{\sqrt{R_{l}^{2}-\left(y-\sqrt{3} R_{l}\right)^{2}}} \mathrm{~d} y, \\
& I_{z_{1}}=4 \int_{0}^{(\sqrt{3} / 2) R_{l}} t R_{l} \frac{y^{2}}{\sqrt{R_{l}^{2}-y^{2}}} \mathrm{~d} y+4 \int_{(\sqrt{3} / 2) R_{l}}^{\sqrt{3} R_{l}} t R_{l} \frac{y^{2}}{\sqrt{R_{l}^{2}-\left(\sqrt{3} R_{l}-y\right)^{2}}} \mathrm{~d} y+4 \int_{\sqrt{3} R_{l}}^{\sqrt{3} R_{l}+q} t y^{2} \mathrm{~d} y .
\end{aligned}
$$

Calculating equations (1) and (2) yields

$$
\begin{aligned}
& I_{y_{1}}=\left(\frac{8 \pi}{3}-3 \sqrt{3}\right) t R_{l}^{3}, \\
& I_{z_{1}}=\left(\frac{16 \pi}{3}-9 \sqrt{3}\right) t R_{l}^{3}+\frac{4}{3} t\left(\sqrt{3} R_{l}+q\right)^{3} .
\end{aligned}
$$

The calculation method referred in [29] is used to examine the equivalent torsional moment of area for the CTM boom, and the following result is obtained:

$$
J_{1}=\frac{18}{\pi} R_{l}^{3} t
$$

Besides, the cross-sectional area of the CTM boom, denoted as $A_{1}$, is given by

$$
A_{1}=\frac{8}{3} \pi t R_{l}+4 q t
$$

\section{Equivalent Mathematical Model of Central Mast}

3.1. Unit of Central Mast. To offer a foundation for efficient analysis and design of the double-level guyed mast, the equivalent beam model of the central mast is established. The simplified repeating unit of the central mast shown in Figure 3 


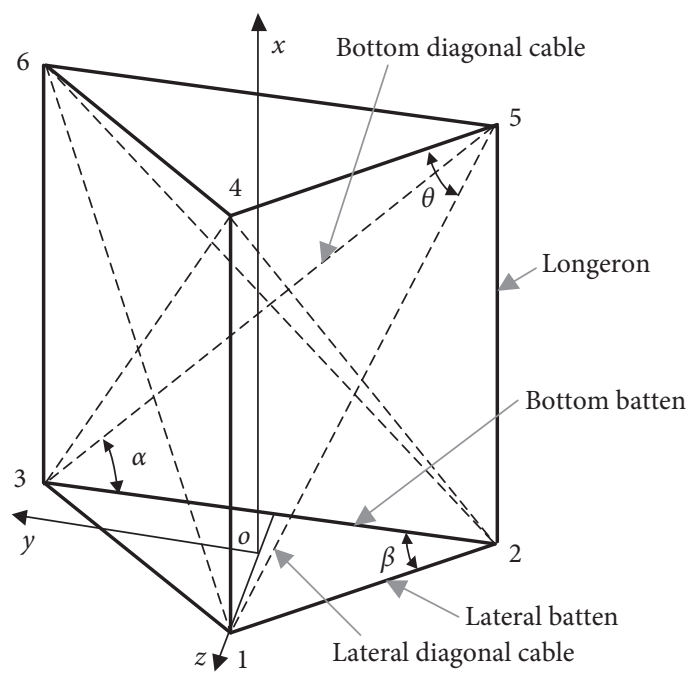

FIgURE 3: Repeating unit of the central mast.

consists of two bottom battens, four lateral battens, three longerons, two bottom diagonal cables, and four lateral diagonal cables. The six joints in the unit are denoted by numbers from 1 to 6 . The origin of the coordinate system is located at the centroid of the bottom triangular frame in the unit. Three types of angles in this unit are defined as follows: the angle between bottom batten and bottom diagonal cable is $\alpha$, the angle between bottom batten and lateral batten is $\beta$, and the angle between lateral batten and lateral diagonal cable is $\theta$.

If the length of each element in the unit is denoted with its two joint numbers, such that $l_{12}$ indicates the length of the batten between joints 1 and 2, there will be five different lengths, respectively, denoted as $l_{1}, l_{2}, l_{3}, l_{4}$, and $l_{5}$ in this unit, and the following equality relations can be concluded:

$$
\left\{\begin{array}{l}
l_{14}=l_{25}=l_{36}=l_{1}, \\
l_{12}=l_{13}=l_{45}=l_{46}=l_{2}, \\
l_{23}=l_{56}=l_{3}, \\
l_{15}=l_{24}=l_{34}=l_{16}=l_{4}, \\
l_{35}=l_{26}=l_{5} .
\end{array}\right.
$$

Furthermore, the relations among parameters of lengths and angles can be described by

$$
l_{1}=l_{2} \tan \theta=l_{3} \tan \alpha=l_{4} \sin \theta=l_{5} \sin \alpha .
$$

The displacement components of the repeating mast unit are expressed as follows.

$$
\left\{\begin{array}{l}
u(x, y, z)=u^{0}(x)-y \phi_{z}^{0}(x)+z \phi_{y}^{0}(x) \\
v(x, y, z)=v^{0}(x)+y \varepsilon_{y}^{0}(x)+z\left(-\phi_{x}^{0}(x)+\frac{1}{2} \gamma_{y z}^{0}(x)\right) \\
w(x, y, z)=w^{0}(x)+z \varepsilon_{z}^{0}(x)+y\left(\phi_{x}^{0}(x)+\frac{1}{2} \gamma_{y z}^{0}(x)\right)
\end{array}\right.
$$

where $u^{0}(x), v^{0}(x)$, and $w^{0}(x)$ are the displacement components at $y=z=0 ; \phi_{x}^{0}(x), \phi_{y}^{0}(x)$, and $\phi_{z}^{0}(x)$ are the rotation components; $\varepsilon_{y}^{0}(x)$ and $\varepsilon_{z}^{0}(x)$ are the extensional strains in the $y$ and $z$ directions; and $\gamma_{y z}^{0}(x)$ is the shearing strain in the plane of the cross section. Based on equation (8), the displacement components of an arbitrary point of the repeating unit are expanded in a Taylor series in the $x$ direction, thus obtaining equation (9) if the derivative terms of strain are ignored:

$$
\left\{\begin{array}{l}
u(x, y, z) \approx u^{0}+x \varepsilon_{x}^{0}-y\left(\phi_{z}^{0}+x \kappa_{y}^{0}\right)+z\left(\phi_{y}^{0}+x \kappa_{z}^{0}\right) \\
v(x, y, z) \approx v^{0}+x\left(\phi_{z}^{0}+\gamma_{x y}^{0}\right)+y \varepsilon_{y}^{0}+z\left(-\phi_{x}^{0}-x \kappa_{x}^{0}+\frac{1}{2} \gamma_{y z}^{0}\right)+\frac{1}{2} x^{2} \kappa_{y}^{0} \\
w(x, y, z) \approx w^{0}+x\left(\gamma_{x y}^{0}-\phi_{y}^{0}\right)+z \varepsilon_{z}^{0}+y\left(\phi_{x}^{0}+x \kappa_{x}^{0}+\frac{1}{2} \gamma_{y z}^{0}\right)-\frac{1}{2} x^{2} \kappa_{z}^{0}
\end{array}\right.
$$

where $\kappa_{x}^{0}$ is the twisting curvature and $\kappa_{y}^{0}$ and $\kappa_{z}^{0}$ are the bending curvatures. Then, the following equations can be obtained: 


$$
\begin{gathered}
\kappa_{y}^{0}=\frac{\partial^{2} v}{\partial x^{2}} \\
\kappa_{z}^{0}=-\frac{\partial^{2} w}{\partial x^{2}}, \\
\kappa_{x}^{0}=\frac{\partial \phi_{x}}{\partial x} .
\end{gathered}
$$

Based on the kinematic assumptions, the strain components have a linear variation in the $y$ and $z$ directions as follows:

$$
\left\{\begin{array}{l}
\varepsilon_{x}=\varepsilon_{x}^{0}-y \kappa_{y}^{0}+z \kappa_{z}^{0}, \\
\varepsilon_{y}=\varepsilon_{y}^{0}, \\
\varepsilon_{z}=\varepsilon_{z}^{0} \\
\gamma_{x y}=\gamma_{x y}^{0}+y \partial \varepsilon_{y}^{0}+z\left(-\kappa_{x}^{0}+\frac{1}{2} \partial \gamma_{y z}^{0}\right), \\
\gamma_{x z}=\gamma_{x z}^{0}+z \partial \varepsilon_{z}^{0}+y\left(\kappa_{x}^{0}+\frac{1}{2} \partial \gamma_{y z}^{0}\right), \\
\gamma_{y z}=\gamma_{y z}^{0},
\end{array}\right.
$$

where $\varepsilon_{x}^{0}$ is the extensional strain of the centerline; $\kappa_{x}^{0}$ is the twist in the $x$ direction; $\kappa_{y}^{0}$ and $\kappa_{z}^{0}$ are the curvature changes in the $y$ and $z$ directions; and $\gamma_{x y}^{0}, \gamma_{x z}^{0}$, and $\gamma_{y z}^{0}$ are the transverse shear strains.

The axial strain of the batten, longeron, and cable elements in the repeating unit can be expressed as follows:

$$
\begin{aligned}
\varepsilon^{(k)}= & \varepsilon_{x}^{(k)} l^{(k)^{2}}+\varepsilon_{y}^{(k)} m^{(k)^{2}}+\varepsilon_{z}^{(k)} n^{(k)^{2}}+\gamma_{y z}^{(k)} m^{(k)} n^{(k)} \\
& +\gamma_{x z}^{(k)} l^{(k)} n^{(k)}+\gamma_{x y}^{(k)} l^{(k)} m^{(k)}
\end{aligned}
$$

where $\varepsilon^{(k)}$ is the axial strain of the $k$ th element; $l^{(k)}, m^{(k)}$, and $n^{(k)}$ are the direction cosines of the axes of the $k$ th element; and $\varepsilon_{x}^{(k)}, \varepsilon_{y}^{(k)}, \varepsilon_{z}^{(k)}, \gamma_{y z}^{(k)}, \gamma_{x z}^{(k)}$, and $\gamma_{x y}^{(k)}$ are the strain components of the $k$ th element. As a batten belongs to two neighbor units, only one bottom batten and two lateral elements should be included in the number of elements. Accordingly, there are 12 elements in a repeating unit. The strain state of each batten, longeron, and cable element is represented by the strain of the middle point in each corresponding element. If an element connects joints $i$ and $j$, the position vector of its middle point will be denoted as $\mathbf{p}^{(i j)}$. Hence, the position matrix $\mathbf{P}$ of the whole mast unit can be expressed as follows:

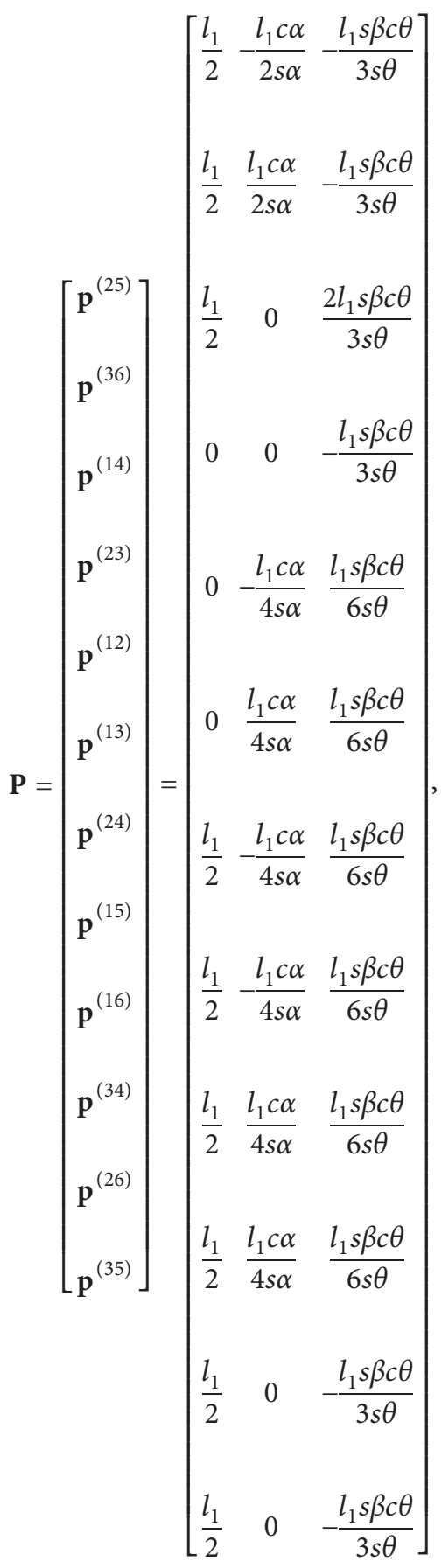

where $s$ represents sin and $c$ represents cos. For the $k$ th element, its direction vector can be expressed by $\left(l(k)^{2}, m^{(k)^{2}}, n^{(k)^{2}}, m^{(k)} n^{(k)}, l^{(k)} n^{(k)}, l^{(k)} m^{(k)}\right)$. Therefore, the corresponding direction matrix $\mathbf{Q}$ to $\mathbf{P}$ can be established with the direction vectors of all elements in the mast unit, which is expressed as follows: 
$$
\mathbf{Q}=\left[\begin{array}{cccccc}
1 & 0 & 0 & 0 & 0 & 0 \\
1 & 0 & 0 & 0 & 0 & 0 \\
1 & 0 & 0 & 0 & 0 & 0 \\
0 & 1 & 0 & 0 & 0 & 0 \\
0 & c^{2} \beta & s^{2} \beta & s \beta c \beta & 0 & 0 \\
0 & c^{2} \beta & s^{2} \beta & -s \beta c \beta & 0 & 0 \\
s^{2} \theta & c^{2} \theta c^{2} \beta & c^{2} \theta s^{2} \beta & c^{2} \theta c \beta s \beta & s \theta c \theta s \beta & s \theta c \theta c \beta \\
s^{2} \theta & c^{2} \theta c^{2} \beta & c^{2} \theta s^{2} \beta & c^{2} \theta c \beta s \beta & -s \theta c \theta s \beta & -s \theta c \theta c \beta \\
s^{2} \theta & c^{2} \theta c^{2} \beta & c^{2} \theta s^{2} \beta & -c^{2} \theta c \beta s \beta & -s \theta c \theta s \beta & s \theta c \theta c \beta \\
s^{2} \theta & c^{2} \theta c^{2} \beta & c^{2} \theta s^{2} \beta & -c^{2} \theta c \beta s \beta & s \theta c \theta s \beta & -s \theta c \theta c \beta \\
s^{2} \alpha & c^{2} \alpha & 0 & 0 & 0 & s \alpha c \alpha \\
s^{2} \alpha & c^{2} \alpha & 0 & 0 & 0 & -s \alpha c \alpha
\end{array}\right] .
$$

With the obtained position matrix and direction matrix, equation (12) is transformed into the following expression:

$$
\varepsilon^{(k)}=\mathbf{I}_{k}\left(\mathrm{AE}_{0}+\mathbf{P K}_{0}\right) \mathbf{Q}^{T} \mathbf{I}_{k}^{T},
$$

where $\mathbf{I}_{k}, A, \mathrm{E}_{0}$, and $\mathrm{K}_{0}$ are shown in the Appendix. In this calculation, the first 3 elements are longerons, the next 3 elements are battens, and the last 6 elements are cables.

3.2. Equivalent Beam. The strain energy of each longeron and batten includes the axial strain energy, the torsion strain energy, and the bending strain energy, whereas that of each cable only includes the axial strain energy. Therefore, the total strain energy of the repeating central mast unit can be expressed as

$$
\begin{aligned}
U_{M}= & \sum_{k=1}^{3} \frac{1}{2} \int_{l_{1}}\left[E_{1} A_{1} \varepsilon^{(k) 2}+E_{1} I_{y_{1}}\left(\frac{\partial^{2} v}{\partial x^{2}}\right)^{2}+E_{1} I_{z_{1}}\left(\frac{\partial^{2} w}{\partial x^{2}}\right)^{2}+G_{1} J_{1}\left(\frac{\partial \phi_{x}}{\partial x}\right)^{2}\right] \mathrm{d} x \\
& +\sum_{k=4}^{6} \frac{1}{2} \int_{l_{1}}\left[E_{2} A_{2} \varepsilon^{(k) 2}+E_{2} I_{y_{2}}\left(\frac{\partial^{2} v}{\partial x^{2}}\right)^{2}+E_{2} I_{z_{2}}\left(\frac{\partial^{2} w}{\partial x^{2}}\right)^{2}+G_{2} J_{2}\left(\frac{\partial \phi_{x}}{\partial x}\right)^{2}\right] \mathrm{d} x \\
& +\sum_{k=7}^{12} \frac{1}{2} \int_{l_{1}} E_{3} A_{3} \varepsilon^{(k) 2} \mathrm{~d} x,
\end{aligned}
$$

where $E_{1} A_{1}, E_{2} A_{2}$, and $E_{3} A_{3}$ denote the axial stiffnesses of the longeron, batten, and beam, respectively; $E_{1} I_{y_{1}}, E_{1} I_{z_{1}}$, $E_{2} I_{y_{2}}$, and $E_{2} I_{z_{2}}$ denote the bending stiffnesses of the longeron and batten in $y$ and $z$ directions, respectively; and $G_{1} J_{1}$ and $G_{2} J_{2}$ denote the torsion stiffnesses of the longeron and batten, respectively. Based on the energy equivalence principle, the total strain energy of the repeating mast unit is equal to that of an anisotropic Timoshenko beam model which is expressed as

$$
U_{B}=\frac{1}{2} \int_{l_{1}} \boldsymbol{\varepsilon}^{T} \mathbf{D} \boldsymbol{\varepsilon} \mathrm{d} x,
$$

where the strain vector $\varepsilon$ and the elasticity matrix $\mathbf{D}$ of the equivalent beam are, respectively, written as

$$
\begin{aligned}
\boldsymbol{\varepsilon} & =\left[\begin{array}{llllll}
\varepsilon_{x}^{0} & \kappa_{y}^{0} & \kappa_{z}^{0} & \kappa_{x}^{0} & \gamma_{x z}^{0} & \gamma_{x y}^{0}
\end{array}\right]^{T}, \\
\mathbf{D} & =\left[\begin{array}{ccccccc}
\overline{E A} & \eta_{12} & \eta_{13} & \eta_{14} & \eta_{15} & \eta_{16} \\
& \overline{E I}_{z} & \eta_{23} & \eta_{24} & \eta_{25} & \eta_{26} \\
& & \overline{E I}_{y} & \eta_{34} & \eta_{35} & \eta_{36} \\
& & & \overline{G J} & \eta_{45} & \eta_{46} \\
& & & & & \overline{G A}_{x z} & \eta_{56} \\
& & & & & & \overline{G A}_{x y}
\end{array}\right],
\end{aligned}
$$

where $\overline{E A}, \overline{E I}_{z}, \overline{E I}_{y}, \overline{G J}, \overline{G A}_{x z}$, and $\overline{G A}_{x y}$ denote the extension, bending, twisting, and shearing stiffnesses of the equivalent beam, and the rest of the matrix elements are the coupling coefficients of the stiffnesses. By comparing the 
corresponding coefficients in the results obtained from equations (16) and (17), the stiffness of the equivalent beam can be solved as

$$
\left\{\begin{array}{l}
\overline{E A}=3 A_{1} E_{1}+2 A_{3} E_{3} \sin ^{4} \alpha+4 A_{3} E_{3} \sin ^{4} \theta, \\
\overline{E I}_{y}=3 E_{1} I_{y_{1}}+\frac{1}{9} A_{3} E_{3} l_{1}^{2} \cos ^{2} \theta \sin ^{2} \theta \sin ^{2} \beta+\frac{2}{3} A_{1} E_{1} l_{1}^{2} \cot ^{2} \theta \sin ^{2} \beta \\
+\frac{2}{9} A_{3} E_{3} l_{1}^{2} \cot ^{2} \theta \sin ^{4} \alpha \sin ^{2} \beta, \\
\overline{E I}_{z}=3 E_{1} I_{z_{1}}+\frac{1}{2} A_{1} E_{1} l_{1}^{2} \cot ^{2} \alpha+\frac{1}{4} A_{3} E_{3} l_{1}^{2} \cot ^{2} \alpha \sin ^{4} \theta, \\
\overline{G J}=3 G_{1} J_{1}+\frac{1}{9} A_{3} E_{3} l_{1}^{2} \cos ^{2} \beta \cos ^{4} \theta \sin ^{2} \beta+\frac{2}{9} A_{3} E_{3} l_{1}^{2} \cos ^{2} \alpha \cot ^{2} \theta \sin ^{2} \alpha \sin ^{2} \beta \\
+\frac{1}{3} A_{3} E_{3} l_{1}^{2} \cos \beta \cos ^{3} \theta \cot ^{2} \alpha \sin ^{2} \beta \sin \theta+\frac{1}{4} A_{3} E_{3} l_{1}^{2} \cos ^{2} \theta \cot ^{2} \alpha \sin ^{2} \beta \sin ^{2} \theta, \\
\overline{G A}_{x z}=4 A_{3} E_{3} \cos ^{2} \theta \sin ^{2} \beta \sin ^{2} \theta, \\
\overline{G A}_{x y}=2 A_{3} E_{3} \cos ^{2} \alpha \sin ^{2} \alpha+4 A_{3} E_{3} \cos ^{2} \beta \cos ^{2} \theta \sin ^{2} \theta .
\end{array}\right.
$$

In this section, the equivalent model of the central mast has been established and will be applied in the static stiffness computation for the equivalent mechanism of the doublelevel guyed mast.

\section{Equivalent Mechanism of Double-Level Guyed Mast}

4.1. Stiffness of SPS Branches. Since the central mast is modeled as an equivalent beam, it is shown as the central beam $\mathrm{O}_{0} \mathrm{O}_{2}$ perpendicular to the base in Figure 4. The triangular frames which the outer and the inner guys are attached to are, respectively, represented as the outer platform and the inner platform. The inner and the outer platforms link with the central beam through spherical joints $O_{1}$ and $\mathrm{O}_{2}$, respectively. The replacement of the tension cable with a SPS kinematic branch is proposed by [12]. The SPS branch consists of two spherical joints and a prismatic joint, which performs the same movement with that of a cable. Therefore, the double-level guyed mast is modeled as a 2(3-SPS-S) mechanism in Figure 4. Coordinate frames $O_{0}-\bar{x}_{0} \bar{y}_{0} \bar{z}_{0}$, $O_{1}-\bar{x}_{1} \bar{y}_{1} \bar{z}_{1}$, and $O_{2}-\bar{x}_{2} \bar{y}_{2} \bar{z}_{2}$ are located at $O_{0}, O_{1}$, and $O_{2}$ on the central beam, respectively. Besides, $\bar{z}_{0}, \bar{z}_{1}$, and $\bar{z}_{2}$ denote the axial direction of the beam. In a SPS mechanism, the anchors on the base, the actuating prismatic joints, and the attachment points to the platform are, respectively, denoted as ${ }^{i} A_{j},{ }^{i} B_{j}$, and ${ }^{i} C_{j}(i=1,2, j=1,2,3)$ in which $i=1$ means the SPS mechanism represents an inner guy and $i=2$ means it represents an outer guy.

The relations between the velocities of the actuating prismatic joints and the platform can be expressed by the velocity Jacobian matrix ${ }^{i} \mathbf{J}_{1}(i=1,2)$ as follows:

$$
{ }^{i} \mathbf{J}_{1}=\left[\begin{array}{cc}
\left({ }^{i} \mathbf{r}_{1}\right)^{T} & \left({ }^{i} \mathbf{e}_{1} \times{ }^{i} \mathbf{r}_{1}\right)^{T} \\
\left({ }^{i} \mathbf{r}_{2}\right)^{T} & \left({ }^{i} \mathbf{e}_{2} \times{ }^{i} \mathbf{r}_{2}\right)^{T} \\
\left({ }^{i} \mathbf{r}_{3}\right)^{T} & \left({ }^{i} \mathbf{e}_{3} \times{ }^{i} \mathbf{r}_{3}\right)^{T}
\end{array}\right]_{3 \times 6},
$$

where ${ }^{i} \mathbf{e}_{j}={ }^{i} C_{j}-O_{i}$ and ${ }^{i} \mathbf{r}_{j}=\left({ }^{i} C_{j}-{ }^{i} A_{j}\right) /\left|{ }^{i} C_{j}-{ }^{i} A_{j}\right|$ $(j=1,2,3)$. If the actuating force and the elastic deformation of the SPS branch are, respectively, denoted as ${ }^{i} F_{a j}$ and $\mathrm{d}^{i} l_{j}$, the stiffness matrix ${ }^{i} \mathbf{K}_{a}$ of the SPS branches can be established as

$$
\left[\begin{array}{c}
{ }^{i} F_{a 1} \\
{ }^{i} F_{a 2} \\
{ }^{i} F_{a 3}
\end{array}\right]={ }^{i} \mathbf{K}_{a}\left[\begin{array}{c}
\mathrm{d}^{i} l_{1} \\
\mathrm{~d}^{i} l_{2} \\
\mathrm{~d}^{i} l_{3}
\end{array}\right],
$$

where ${ }^{i} \mathbf{K}_{a}$ is a $3 \times 3$ symmetric stiffness matrix as follows:

$$
{ }^{i} \mathbf{K}_{a}=\left[\begin{array}{ccc}
{ }^{i} K_{l}\left({ }^{i} l_{1}\right)^{-1} & 0 & 0 \\
0 & { }^{i} K_{l}\left({ }^{i} l_{2}\right)^{-1} & 0 \\
0 & 0 & { }^{i} K_{l}\left({ }^{i} l_{3}\right)^{-1}
\end{array}\right],
$$




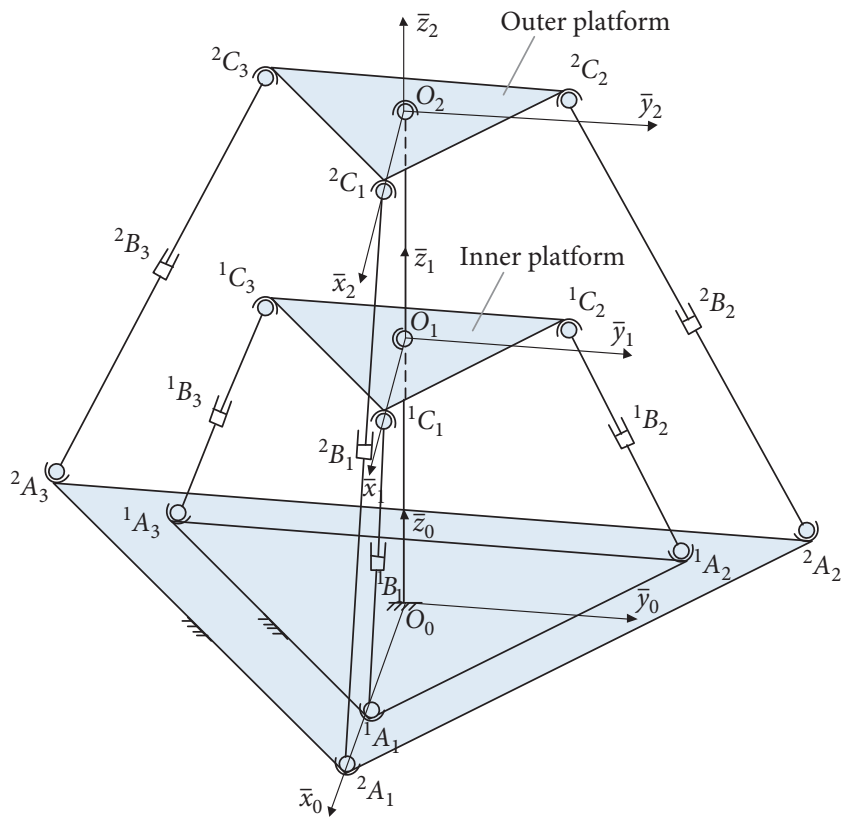

FIGURE 4: Equivalent 2(3-SPS-S) mechanism of the double-level guyed mast.

where ${ }^{i} k_{l}$ is the one-meter stiffness of the guy and ${ }^{i} l_{j}$ is the measured length of the guy. The state of each guy is denoted as ${ }^{i} C s_{j k^{\prime}}$, in which $k$ ' indicates the times of the iteration. When a guy is tensioned, its corresponding ${ }^{i} C s_{j k^{\prime}}$ is 1 ; otherwise is 0 . If ${ }^{i} s_{k^{\prime}}=\operatorname{diag}\left[{ }^{i} C s_{1 k^{\prime}}{ }^{i} C s_{2 k^{\prime}}{ }^{i} C s_{3 k^{\prime}}\right]$ expresses the state of each level of guys, the stiffness matrix in the $k$ 'th iteration can be written as

$$
{ }^{i} \bar{K}_{a}={ }^{i} \mathbf{K}_{a}{ }^{i} \mathbf{s}_{k^{\prime}} .
$$

4.2. Stiffness Analysis of 2(3-SPS-S) Mechanism. The load state of the central beam is shown in Figure 5. The distance between $O_{0}$ and $O_{1}$ is $d_{1}$, and that between $O_{1}$ and $O_{2}$ is $d_{2}$. The external loads acting on the beam at $O_{i}$ of the central beam are denoted as $\mathbf{T}_{i}$, which can be expressed as

$$
\mathbf{T}_{i}=\left[{ }^{i} F_{x}{ }^{i} F_{y} \quad{ }^{i} F_{z}{ }^{i} M_{x}{ }^{i} M_{y}{ }^{i} M_{z}\right]^{T} .
$$

Meanwhile, $\delta_{i}$ representing the displacement at $O_{i}$ is expressed as

$$
\boldsymbol{\delta}_{i}=\left[\begin{array}{llllll}
{ }^{i} \omega_{x} & { }^{i} \omega_{y} & { }^{i} \omega_{z} & { }^{i} \theta_{x} & { }^{i} \theta_{y} & { }^{i} \theta_{z}
\end{array}\right]^{T} .
$$

The elastic deformations in each level of SPS branches can hence be written as

$$
\left[\begin{array}{l}
\mathrm{d}^{i} l_{1} \\
\mathrm{~d}^{i} l_{2} \\
\mathrm{~d}^{i} l_{3}
\end{array}\right]={ }^{i} \mathbf{J}_{1} \boldsymbol{\delta}_{i} .
$$

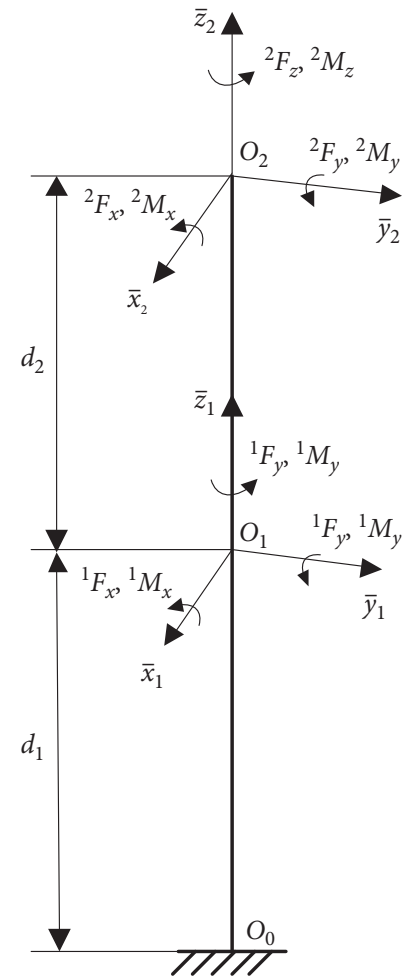

Figure 5: Load state of the central beam.

Besides, based on the obtained results in Section 3, the stiffness parameters of this equivalent central beam in the 2(3-SPS-S) mechanism can be expressed as

$$
\begin{aligned}
E A & =\overline{E A}, \\
E I_{x} & =\overline{E I}_{z}, \\
E I_{y} & =\overline{E I}_{y}, \\
G J & =\overline{G J} .
\end{aligned}
$$

As the central mast has been modeled as an anisotropic beam, the load analysis of the central beam is carried out in different directions. The load states of the central beam in $x$ and $y$ directions are shown in Figure 6. The distance from $O_{0}$ to any point between $O_{0}$ and $O_{1}$ is denoted as $s_{1}$, while that from $O_{0}$ to any point between $O_{1}$ and $O_{2}$ is denoted as $s_{2}$. Based on the definition of $s_{1}$ and $s_{2}$, their ranges can be written as follows.

$$
\begin{aligned}
& s_{1} \in\left[0, d_{1}\right], \\
& s_{2} \in\left[d_{1},\left(d_{1}+d_{2}\right)\right] .
\end{aligned}
$$

The forces and moments in different directions for a point positioned with $s_{i}(i=1,2)$ are denoted as $F_{x i}, F_{y i}, F_{z i}$, $M_{x i}, M_{y i}$, and $M_{z i}$ while the deflections in $x$ and $y$ directions for this point are denoted as $\omega_{x i}$ and $\omega_{y i}$. According to the load states shown in Figure 6, the moments in the beam can be calculated as follows: 


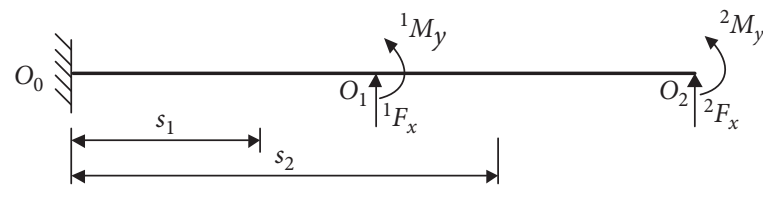

(a)

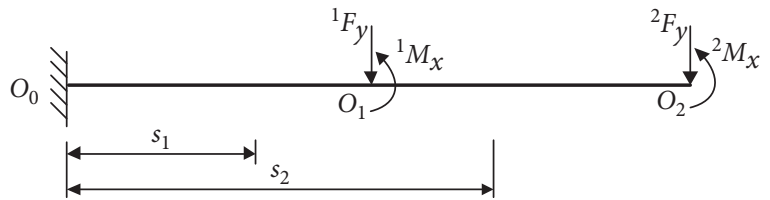

(b)

FIgURE 6: Load states of the central beam: (a) in $x$ direction and (b) in $y$ direction.

$$
\left\{\begin{array}{l}
E I_{x} \omega_{y 1}^{\prime \prime}\left(s_{1}\right)=M_{x 1}\left(s_{1}\right)={ }^{1} M_{x}+{ }^{2} M_{x}-{ }^{1} F_{y}\left(d_{1}-s_{1}\right)-{ }^{2} F_{y}\left(d_{1}+d_{2}-s_{1}\right), \\
E I_{y} w_{x 1}^{\prime \prime}\left(s_{1}\right)=M_{y 1}\left(s_{1}\right)={ }^{1} M_{y}+{ }^{2} M_{y}+{ }^{1} F_{x}\left(d_{1}-s_{1}\right)+{ }^{2} F_{x}\left(d_{1}+d_{2}-s_{1}\right), \\
E I_{x} \omega_{y 2}^{\prime \prime}\left(s_{2}\right)=M_{x 2}\left(s_{2}\right)={ }^{2} M_{x}-{ }^{2} F_{y}\left(d_{1}+d_{2}-s_{2}\right), \\
E I_{y} w_{x 2}^{\prime \prime}\left(s_{2}\right)=M_{y 2}\left(s_{2}\right)={ }^{2} M_{y}+{ }^{2} F_{x}\left(d_{1}+d_{2}-s_{2}\right) .
\end{array}\right.
$$

Besides, the forces and moments in the $z$ direction of the beam can be obtained as follows:

$$
\left\{\begin{array}{l}
F_{z 1}\left(s_{1}\right)={ }^{1} F_{z}+{ }^{2} F_{z}, \\
M_{z 1}\left(s_{1}\right)={ }^{1} M_{z}+{ }^{2} M_{z}, \\
F_{z 2}\left(s_{2}\right)={ }^{2} F_{z}, \\
M_{z 2}\left(s_{2}\right)={ }^{2} M_{z} .
\end{array}\right.
$$

As the central beam is a continuous beam constrained by the base, the characteristics of its deflection can offer boundary and continuity conditions for the calculation. The boundary conditions for the central beam are expressed as

$$
\begin{aligned}
s_{1}=0: \omega_{x 1}\left(s_{1}\right) & =0, \\
\omega_{x 1}^{\prime}\left(s_{1}\right) & =0, \\
\omega_{y 1}\left(s_{1}\right) & =0, \\
\omega_{y 1}^{\prime}\left(s_{1}\right) & =0 .
\end{aligned}
$$

Meanwhile, the continuity conditions for the central beam are expressed as

$$
\begin{aligned}
s_{1}=s_{2}=d_{1}: \omega_{x 1}\left(s_{1}\right) & =\omega_{x 2}\left(s_{2}\right), \\
\omega_{x 1}^{\prime}\left(s_{1}\right) & =\omega_{x 2}^{\prime}\left(s_{2}\right), \\
\omega_{y 1}\left(s_{1}\right) & =\omega_{y 2}\left(s_{2}\right), \\
\omega_{y 1}^{\prime}\left(s_{1}\right) & =\omega_{y 2}^{\prime}\left(s_{2}\right) .
\end{aligned}
$$

The formula of force deformation can be derived from equations (29) and (30) with the condition equations, and it is solved as

$$
\left[\begin{array}{l}
\mathbf{T}_{1} \\
\mathbf{T}_{2}
\end{array}\right]=\left[\begin{array}{ll}
\mathbf{K}_{11} & \mathbf{K}_{12} \\
\mathbf{K}_{21} & \mathbf{K}_{22}
\end{array}\right]^{-1}\left[\begin{array}{l}
\boldsymbol{\delta}_{1} \\
\boldsymbol{\delta}_{2}
\end{array}\right],
$$

where $\mathbf{K}_{11}, \mathbf{K}_{12}, \mathbf{K}_{21}$, and $\mathbf{K}_{22}$ are shown in the Appendix. Transforming equation (33) yields the following expression:

$$
\left[\begin{array}{l}
\mathbf{T}_{1} \\
\mathbf{T}_{2}
\end{array}\right]=\left[\begin{array}{ll}
\overline{\mathbf{K}}_{11} & \overline{\mathbf{K}}_{12} \\
\overline{\mathbf{K}}_{21} & \overline{\mathbf{K}}_{22}
\end{array}\right]\left[\begin{array}{l}
\boldsymbol{\delta}_{1} \\
\boldsymbol{\delta}_{2}
\end{array}\right],
$$

where $\overline{\mathbf{K}}_{11}, \overline{\mathbf{K}}_{12}, \overline{\mathbf{K}}_{21}$, and $\overline{\mathbf{K}}_{22}$ are shown in the Appendix. Afterwards, the expressions of $\mathbf{T}_{1}$ and $\mathbf{T}_{2}$ are revealed as

$$
\begin{aligned}
& \mathbf{T}_{1}=\overline{\mathbf{K}}_{11} \boldsymbol{\delta}_{1}+\overline{\mathbf{K}}_{12} \boldsymbol{\delta}_{2}, \\
& \mathbf{T}_{2}=\overline{\mathbf{K}}_{21} \boldsymbol{\delta}_{1}+\overline{\mathbf{K}}_{22} \boldsymbol{\delta}_{2} .
\end{aligned}
$$

Based on the principle of virtual work, the following equation exists at $O_{1}$ :

$$
\boldsymbol{\delta}_{1}^{T} \mathbf{T}_{1}+\left[\begin{array}{l}
\mathrm{d}^{1} l_{1} \\
\mathrm{~d}^{1} l_{2} \\
\mathrm{~d}^{1} l_{3}
\end{array}\right]^{T}\left[\begin{array}{c}
{ }^{1} F_{a 1} \\
{ }^{1} F_{a 2} \\
{ }^{1} F_{a 3}
\end{array}\right]=0 .
$$

Substituting equations (21) and (26) into (37) yields

$$
\mathbf{T}_{1}=-\left({ }^{1} \mathbf{J}_{1}\right)^{T}{ }^{T} \overline{\mathbf{K}}_{1}{ }^{1} \mathbf{J}_{1} \boldsymbol{\delta}_{1} .
$$

Substituting equation (38) into (35) leads to

$$
\boldsymbol{\delta}_{1}=\overline{\mathbf{K}} \boldsymbol{\delta}_{2},
$$

where

$$
\overline{\mathbf{K}}=-\left[\overline{\mathbf{K}}_{11}+\left({ }^{1} \mathbf{J}_{1}\right)^{T} \bar{K}_{a}^{1} \mathbf{J}_{1}\right]^{-1} \overline{\mathbf{K}}_{12} .
$$

Introducing equation (39) into (36) yields the following expression of $\mathbf{T}_{2}$ with $\delta_{2}$ :

$$
\mathbf{T}_{2}=\left(\overline{\mathbf{K}}_{21} \overline{\mathbf{K}}+\overline{\mathbf{K}}_{22}\right) \boldsymbol{\delta}_{2} .
$$

Assuming the load applied to the whole 2(3-SPS-S) mechanism on the outer platform is denoted as $\mathbf{T}$, the following equation can be established according to the principle of virtual work:

$$
\left[\begin{array}{l}
\mathrm{d}^{2} l_{1} \\
\mathrm{~d}^{2} l_{2} \\
\mathrm{~d}^{2} l_{3}
\end{array}\right]^{T}\left[\begin{array}{c}
{ }^{2} F_{a 1} \\
{ }^{2} F_{a 2} \\
{ }^{2} F_{a 3}
\end{array}\right]+\boldsymbol{\delta}_{2}^{T} \mathbf{T}_{2}=\boldsymbol{\delta}_{2}^{T} \mathbf{T} .
$$


The above equation can be rewritten by introducing equations (21) and (26) into (42) as

$$
\boldsymbol{\delta}_{2}^{T}\left({ }^{2} \mathbf{J}_{1}\right)^{T} \bar{K}_{a}\left({ }^{2} \mathbf{J}_{1}\right) \boldsymbol{\delta}_{2}+\boldsymbol{\delta}_{2}^{T} \mathbf{T}_{2}=\boldsymbol{\delta}_{2}^{T} \mathbf{T}
$$

Substituting the expression of $\mathbf{T}_{2}$ in equation (41) into the above presents

$$
\mathbf{T}=\mathbf{K} \boldsymbol{\delta}_{2}
$$

where

$$
\mathbf{K}=\overline{\mathbf{K}}_{21} \overline{\mathbf{K}}+\overline{\mathbf{K}}_{22}+\left({ }^{2} \mathbf{J}_{1}\right)^{T} \bar{K}_{a}\left({ }^{2} \mathbf{J}_{1}\right) .
$$

In this section, the total stiffness matrix of the equivalent 2(3-SPS-S) mechanism for the double-level guyed mast is finally obtained, which establishes the relation between the load and deformation at the end of the central mast.

\section{Analysis of Static Stiffness of Double-Level Guyed Mast}

Based on the theoretical modeling of the double-level guyed mast in Figure 4, the static stiffness of the double-level guyed mast can be examined. In a membrane antenna, the bending stiffness of the guyed mast mainly affects the accuracy of a planar membrane. Therefore, the distribution of guys should satisfy the optimal bending stiffness. The ratios of load to deformation in the $x$ and $y$ directions are denoted as the static stiffnesses $K_{x}$ and $K_{y}$, respectively. In this section, the analysis is based on a double-level guyed mast in which some parameters of the central mast have been assigned. The length of each mast unit is $2.5 \mathrm{~m}$, and the total length of the guyed mast is denoted as $l_{m}$. In the isosceles triangular cross section of the central mast, $b_{0}$ is $3 \mathrm{~m}$, and $h_{0}$ is $1 \mathrm{~m}$. Each guy should be tensioned when the structure deforms, and the tension force is set as $100 \mathrm{~N}$. On the base, the anchors of the inner guys form an isosceles triangle whose width and height are denoted as $b_{1}$ and $h_{1}$, respectively. Meanwhile, the width and height of the isosceles triangle formed by the anchors of the outer guys are denoted as $b_{2}$ and $h_{2}$, respectively. The radius of CTM is denoted as $R_{l}$, and the radius of guys is denoted as $R_{g}$. Besides, the material and sectional parameters of the mast elements are shown in Table 1.

Considering the size of the base is limited in space, $b_{2}$ and $h_{2}$ are set as $9 \mathrm{~m}$ and $3 \mathrm{~m}$, respectively, whereas $b_{1}, h_{1}$, and $d_{1}$ are set as variables. The ratio of $b_{1}$ to $h_{1}$ is set as 3 , which is the same as that of $b_{0}$ to $h_{0}$. Besides, $l_{m}$ is set as $30 \mathrm{~m}$. Under these conditions, the influences of $d_{1}$ and $h_{1}$ on the static stiffnesses $K_{x}$ and $K_{y}$ are investigated and shown in Figures 7 and 8, respectively. It is found that increasing $h_{1}$ can raise both $K_{x}$ and $K_{y}$. Moreover, $K_{x}$ and $K_{y}$ will both reach a peak when $d_{1}$ is around $25 \mathrm{~m}$. When $d_{1}$ is larger than $25 \mathrm{~m}, K_{x}$ and $K_{y}$ will both decrease slightly. Therefore, $d_{1}$ and $h_{1}$ should be, respectively, set as $25 \mathrm{~m}$ and $3 \mathrm{~m}$ for the optimal static stiffness of the double-level guyed mast. In this situation, $h_{1}$ is equal to $h_{2}$, and $b_{1}$ is equal to $b_{2}$.

For the given base in which the width and height are, respectively, set as $9 \mathrm{~m}$ and $3 \mathrm{~m}$, the optimal distribution of guys is shown in Figure 9. The anchors of the inner guys coincide with those of outer guys. The parameter $d_{2}$ is $5 \mathrm{~m}$, which means there are two mast units between the inner and the outer platforms.

If the above optimized configuration is designated as Case 1, other cases can be obtained by changing each parameter of Case 1. Five feature parameters are chosen in a case, including $R_{g}, h_{1}, R_{l}, l_{m}$, and $d_{1}$. Therefore, the six different cases can be established as follows.

$$
\begin{aligned}
& \text { Case 1: } R_{g}=2 \mathrm{~mm}, R_{l}=80 \mathrm{~mm}, h_{1}=3 \mathrm{~m}, l_{m}=30 \mathrm{~m}, \\
& d_{1}=25 \mathrm{~m} \\
& \text { Case 2: } R_{g}=3 \mathrm{~mm}, R_{l}=80 \mathrm{~mm}, h_{1}=3 \mathrm{~m}, l_{m}=30 \mathrm{~m}, \\
& d_{1}=25 \mathrm{~m} \\
& \text { Case 3: } R_{g}=2 \mathrm{~mm}, R_{l}=100 \mathrm{~mm}, h_{1}=3 \mathrm{~m}, l_{m}=30 \mathrm{~m}, \\
& d_{1}=25 \mathrm{~m} \\
& \text { Case } 4: R_{g}=2 \mathrm{~mm}, R_{l}=80 \mathrm{~mm}, h_{1}=4 \mathrm{~m}, l_{m}=30 \mathrm{~m}, \\
& d_{1}=25 \mathrm{~m} \\
& \text { Case } 5: R_{g}=2 \mathrm{~mm}, R_{l}=80 \mathrm{~mm}, h_{1}=3 \mathrm{~m}, l_{m}=40 \mathrm{~m}, \\
& d_{1}=25 \mathrm{~m} \\
& \text { Case } 6: R_{g}=2 \mathrm{~mm}, R_{l}=80 \mathrm{~mm}, h_{1}=3 \mathrm{~m}, l_{m}=30 \mathrm{~m}, \\
& d_{1}=15 \mathrm{~m}
\end{aligned}
$$

To verify the theoretical model of 2(3-SPS-S) mechanism shown in Figure 4, its results are compared with those obtained from finite element analysis (FEA) model. In the FEA model, the ratios of load to deformation in the $x$ and $y$ directions are denoted as $K_{x}^{*}$ and $K_{y}^{*}$, respectively. Three equal loads are applied at the three end nodes of the central mast, respectively. The deformation of the guyed mast is indicated by the average displacement of the three end nodes. The coordinate system used to measure the results is $\mathrm{O}_{2}-\bar{x}_{2} \bar{y}_{2} \bar{z}_{2}$ as shown in Figure 9, which is for both FEA model and theoretical model. In addition, the errors between the results of FEA model and theoretical model are denoted as $\xi_{x}$ and $\xi_{y}$, respectively, which are expressed as follows:

$$
\begin{aligned}
& \xi_{x}=\frac{K_{x}-K_{x}^{*}}{K_{x}^{*}} \times 100 \%, \\
& \xi_{y}=\frac{K_{y}-K_{y}^{*}}{K_{y}^{*}} \times 100 \% .
\end{aligned}
$$

The comparison of the results from FEA model and theoretical model is shown in Table 2, and it is found that the errors are acceptable. In particular, the accuracy of the theoretical model in the $x$ direction is higher than that in the $y$ direction. Generally, the equivalent mechanism is reliable enough for the purpose of pre-design.

Further, the Genetic Algorithm (GA) is used to conduct the optimization of the static stiffness of the double-level guyed mast. According to the above comparison, $K_{x}$ is more accurate than $K_{y}$, and $K_{x}$ performs the same variation regularity with $K_{y}$ as shown in Figures 7 and 8. Therefore, $K_{x}$ is chosen in the optimization problem which can be established as follows. 
TABLE 1: Material and sectional parameters of the mast elements.

\begin{tabular}{lccc}
\hline Items & Battens & Diagonal cables & CTM \\
\hline Material & Carbon fiber & Kevlar & Composite \\
Elasticity modulus $E(\mathrm{GPa})$ & 350 & 131 & 114 \\
Density $\rho\left(\mathrm{kg} / \mathrm{m}^{3}\right)$ & 2000 & 1435 & 1600 \\
Poisson's ratio $v$ & 0.30 & 0.30 & 0.30 \\
\hline
\end{tabular}

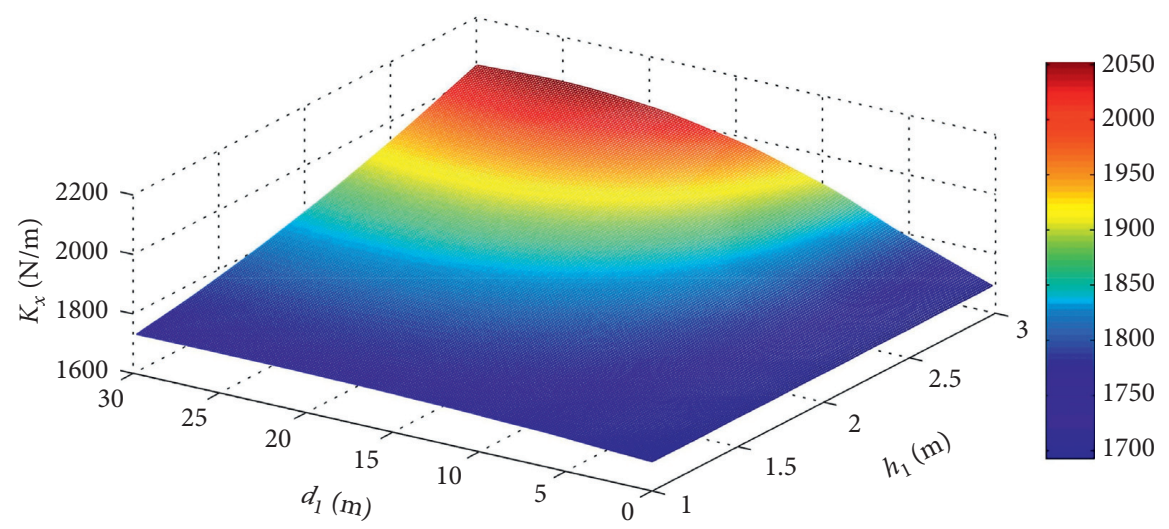

FIGURE 7: Influence of $d_{1}$ and $h_{1}$ on $K_{x}$.

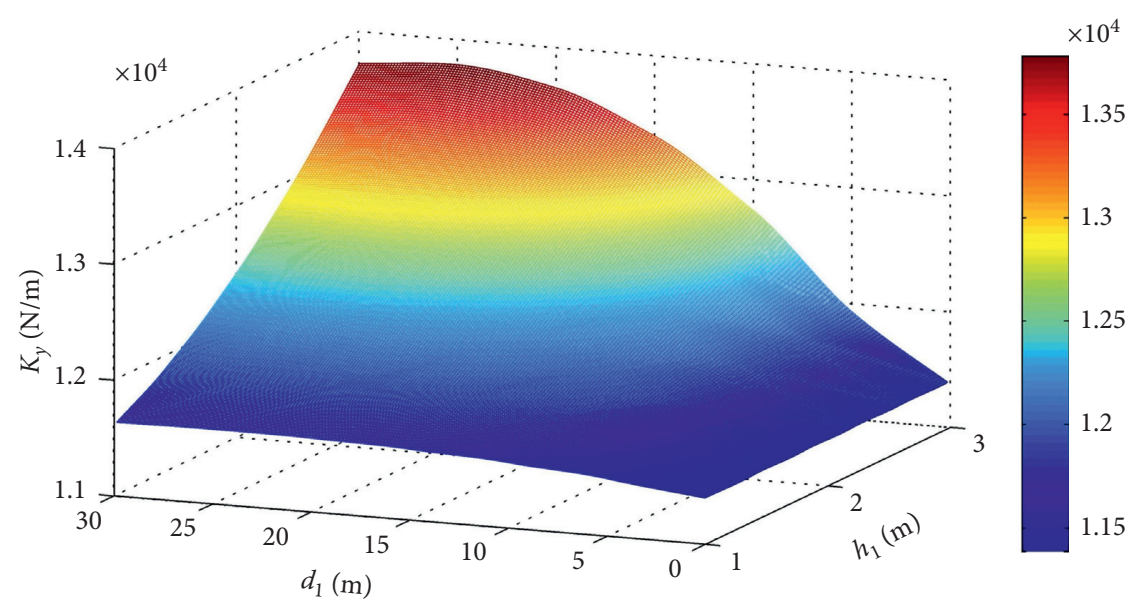

Figure 8: Influence of $d_{1}$ and $h_{1}$ on $K_{y}$.

Find $\chi=\left(h_{1}, b_{1}, d_{1}\right)^{T}$,

$$
\begin{array}{ll}
\operatorname{Min} & \Psi(\chi)=\frac{1}{K_{x}} \\
\text { s.t. } \quad\left\{\begin{array}{l}
b_{1}=3 h_{1}, \\
h_{0} \leq h_{1} \leq h_{2}, \\
0 \leq d_{1} \leq l_{m} .
\end{array}\right.
\end{array}
$$

Based on equation (47), the optimization results calculated with GA show $h_{1}$ is equal to $h_{2}$, whereas the optimal $d_{1}$ is related to $l_{m}$. In addition, $\eta$ is the ratio of $d_{1}$ to $l_{m}$, which is going to be investigated in different parametric conditions. The effects from $R_{g}, R_{l}$, and $h_{2}$ on the optimal $\eta$ are studied and presented in Figures 10-12, respectively. When the length of the guyed mast increases, the optimal $\eta$ decreases in a nonlinear pattern but finally infinitely approaches a constant value. It is revealed in Figure 10 that the larger the radius of guys is, the lower the optimal $\eta$ will be. Differently, the larger the radius of the CTM is, the higher the optimal $\eta$ will be obtained, which is depicted in Figure 11. According to Figure 12 , it is noticed that increasing $h_{2}$ can raise the optimal $\eta$ as well. By comparing Figures 10, 11, and 12, it is demonstrated that $R_{g}, R_{l}$, and $h_{2}$ influence the optimal $\eta$ by different means. The variation curves in Figure 10 or 11 tend to be parallel to each other, but those in Figure 12 do not show this characteristic. Besides, when $R_{l}$ is raised to a certain degree, the increase of the optimal $\eta$ is limited. Among these three variation conditions, it is found that changing the radius of guys could be the most efficient way to adjust the optimal $\eta$.

Given a certain length of the guyed mast, the influence of $R_{l}$ on the optimal $d_{1}$ is investigated and presented in 


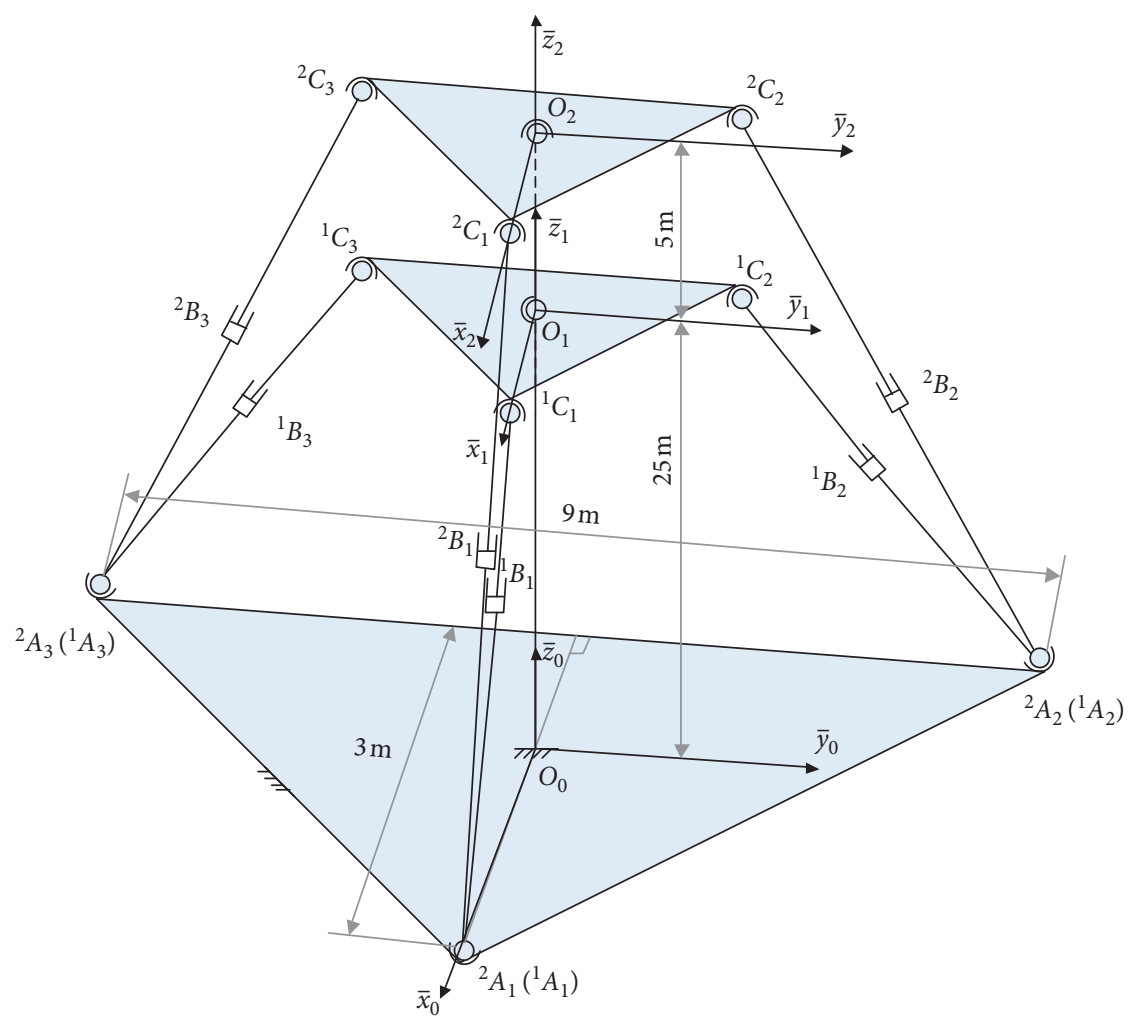

FIgURE 9: Distribution of guys for optimal static stiffness.

TABLE 2: Comparison between the results of FEA model and theoretical model.

\begin{tabular}{llllll}
\hline Cases & $K_{x}^{*}(\mathrm{~N} / \mathrm{m})$ & $K_{x}(\mathrm{~N} / \mathrm{m})$ & $\xi_{x}$ & $K_{y}^{*}(\mathrm{~N} / \mathrm{m})$ & $K_{y}(\mathrm{~N} / \mathrm{m})$ \\
\hline Case 1 & $2.02 \times 10^{3}$ & $2.05 \times 10^{3}$ & $1.49 \%$ & $1.17 \times 10^{4}$ & $1.38 \times 10^{4}$ \\
Case 2 & $2.86 \times 10^{3}$ & $3.04 \times 10^{3}$ & $6.29 \%$ & $1.71 \times 10^{4}$ & $2.04 \times 10^{4}$ \\
Case 3 & $2.27 \times 10^{3}$ & $2.36 \times 10^{3}$ & $3.96 \%$ & $1.36 \times 10^{4}$ & $1.58 \times 10^{4}$ \\
Case 4 & $2.42 \times 10^{3}$ & $2.59 \times 10^{3}$ & $7.02 \%$ & $1.52 \times 10^{4}$ & $1.73 \times 10^{4}$ \\
Case 5 & $8.29 \times 10^{2}$ & $8.57 \times 10^{2}$ & $3.38 \%$ & $5.46 \times 10^{3}$ & $5.76 \times 10^{3}$ \\
Case 6 & $1.96 \times 10^{3}$ & $1.99 \times 10^{3}$ & $1.53 \%$ & $1.15 \times 10^{4}$ & $13.18 \%$ \\
\hline
\end{tabular}

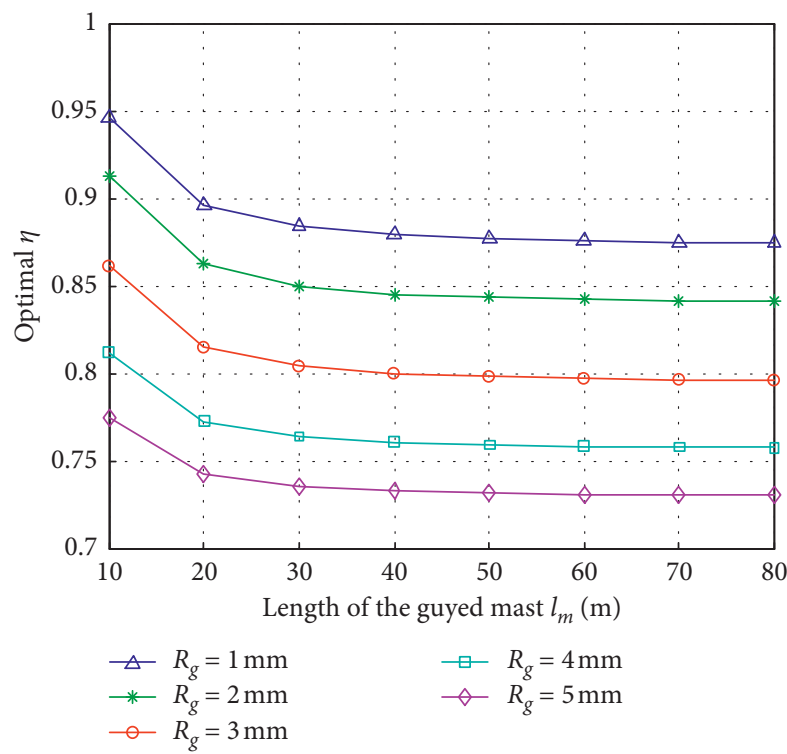

Figure 10: Influence of $R_{g}$ on the optimal $\eta$.

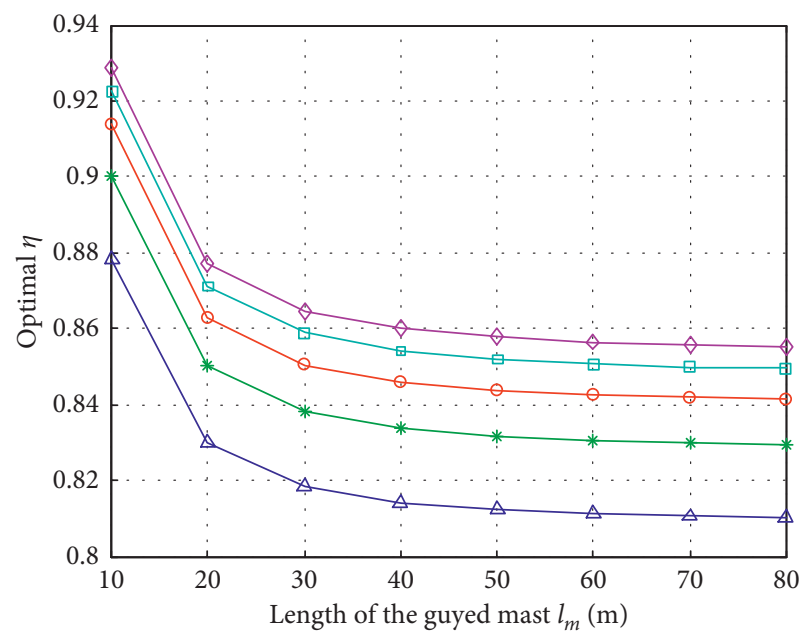

$$
\begin{array}{ll}
\triangle R_{l}=40 \mathrm{~mm} & \rightarrow-R_{l}=100 \mathrm{~mm} \\
\rightarrow-R_{l}=60 \mathrm{~mm} & \multimap-R_{l}=120 \mathrm{~mm} \\
\odot R_{l}=80 \mathrm{~mm} &
\end{array}
$$

Figure 11: Influence of $R_{l}$ on the optimal $\eta$. 


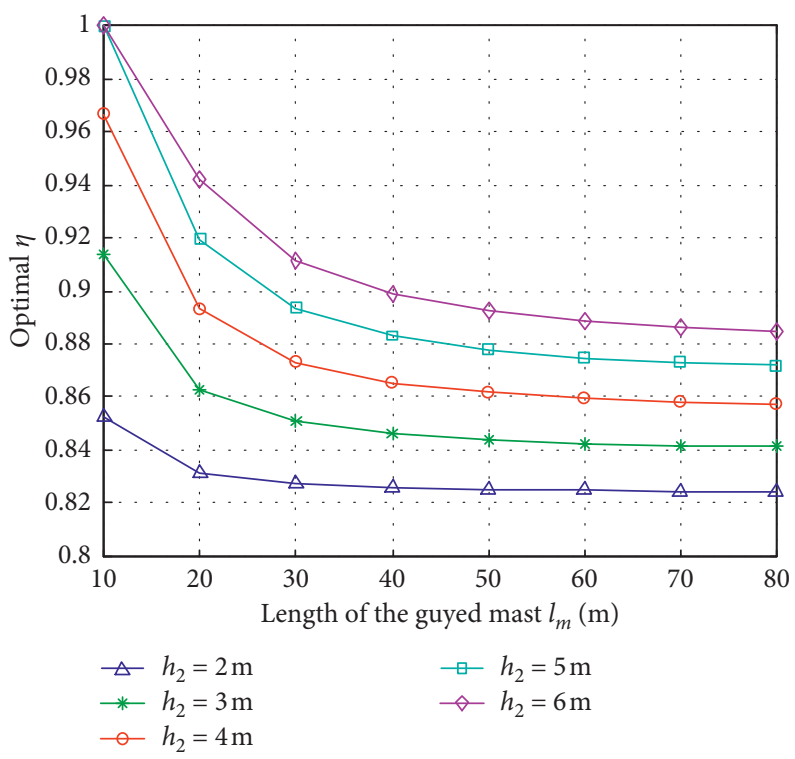

FIGURE 12: Influence of $h_{2}$ on the optimal $\eta$.

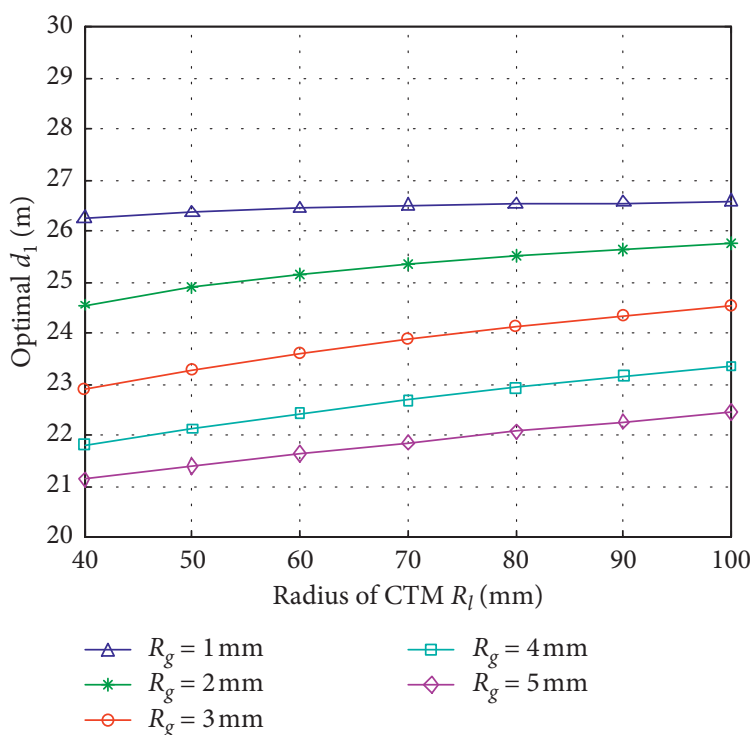

FIgURE 13: Influence of $R_{l}$ on the optimal $d_{1}$.

Figure 13. The length of the guyed mast is set as $30 \mathrm{~m}$, and the radius of guys varies from $1 \mathrm{~mm}$ to $5 \mathrm{~mm}$. When $R_{g}$ is $1 \mathrm{~mm}$, the increase of $R_{l}$ hardly influences the optimal $d_{1}$. Besides, the larger the radius of guys, the more obvious $R_{l}$ affects the optimal $d_{1}$. Therefore, the influencing degree of $R_{l}$ on the optimal $d_{1}$ depends on the thickness of guys.

\section{Conclusions}

This paper presents the stiffness analysis of a double-level guyed mast for a membrane antenna and the optimal distribution of guys for enhancing static stiffness. Based on the energy equivalence principle, the central mast is mathematically modeled as an anisotropic Timoshenko beam. Afterwards, the double-level guyed mast is modeled as a 2(3SPS-S) mechanism in which the stiffnesses of the SPS branches and the central double-level loaded mast are derived, respectively. The total stiffness matrix of the equivalent mechanism is obtained according to the principle of virtual work. The comparison between results of FEA model and theoretical model indicates the equivalent mechanism is reliable enough for the purpose of pre-design.

The influence of distribution of guys on static stiffness is discussed in detail. The anchors of the inner guys should coincide with those of the outer guys for the optimal static stiffness. The Genetic Algorithm is used to conduct the optimization of the static stiffness, which illustrates the influences from $R_{g}, R_{l}$, and $h_{2}$ on the optimal $\eta$. Among these three variation conditions, changing the radius of guys could be the most efficient way to adjust the optimal $\eta$. The analysis and design of the double-level guyed mast 
provides a reference for the equivalence of a multilevel guyed mast.

\section{Appendix}

$$
\begin{aligned}
& \mathbf{I}_{k}=\left[\begin{array}{lllll}
\underbrace{0}_{k-1} \cdots & 1 & \underbrace{0}_{12-k} \cdots
\end{array}\right], \\
& \mathbf{A}=\left[\begin{array}{llllllllllll}
1 & 1 & 1 & 1 & 1 & 1 & 1 & 1 & 1 & 1 & 1 & 1
\end{array}\right]^{T}, \\
& \mathrm{E}_{0}=\left[\begin{array}{llllll}
\varepsilon_{x}^{0} & \varepsilon_{y}^{0} & \varepsilon_{z}^{0} & \gamma_{y z}^{0} & \gamma_{x z}^{0} & \gamma_{x y}^{0}
\end{array}\right], \\
& \mathrm{K}_{0}=\left[\begin{array}{cccccc}
0 & 0 & 0 & 0 & 0 & 0 \\
-\kappa_{y}^{0} & 0 & 0 & 0 & \kappa_{x}^{0} & 0 \\
\kappa_{z}^{0} & 0 & 0 & 0 & 0 & -\kappa_{x}^{0}
\end{array}\right], \\
& \mathbf{K}_{11}=\left[\begin{array}{cccccc}
\frac{d_{1}^{3}}{3 E I_{y}} & 0 & 0 & 0 & \frac{d_{1}^{2}}{2 E I_{y}} & 0 \\
0 & \frac{d_{1}^{3}}{3 E I_{x}} & 0 & -\frac{d_{1}^{2}}{2 E I_{x}} & 0 & 0 \\
0 & 0 & \frac{d_{1}}{E A} & 0 & 0 & 0 \\
\frac{d_{1}^{2}}{2 E I_{y}} & 0 & 0 & 0 & \frac{d_{1}}{E I_{y}} & 0 \\
0 & \frac{d_{1}^{2}}{2 E I_{x}} & 0 & -\frac{d_{1}}{E I_{x}} & 0 & 0 \\
0 & 0 & 0 & 0 & 0 & \frac{d_{1}}{G J}
\end{array}\right] \text {, } \\
& \mathbf{K}_{12}=\left[\begin{array}{cccccc}
\frac{d_{1}^{2}\left(2 d_{1}+3 d_{2}\right)}{6 E I_{y}} & 0 & 0 & 0 & \frac{d_{1}^{2}}{2 E I_{y}} & 0 \\
0 & \frac{d_{1}^{2}\left(2 d_{1}+3 d_{2}\right)}{6 E I_{x}} & 0 & -\frac{d_{1}^{2}}{2 E I_{x}} & 0 & 0 \\
0 & 0 & \frac{d_{1}}{E A} & 0 & 0 & 0 \\
\frac{d_{1}\left(d_{1}+2 d_{2}\right)}{2 E I_{y}} & 0 & 0 & 0 & \frac{d_{1}}{E I_{y}} & 0 \\
0 & \frac{d_{1}\left(d_{1}+2 d_{2}\right)}{2 E I_{x}} & 0 & -\frac{d_{1}}{E I_{x}} & 0 & 0 \\
0 & 0 & 0 & 0 & 0 & \frac{d_{1}}{G J}
\end{array}\right] \text {, }
\end{aligned}
$$




$$
\begin{aligned}
& \mathbf{K}_{21}=\left[\begin{array}{cccccc}
\frac{2 d_{1}^{3}+3 d_{1}^{2} d_{2}}{6 E I_{y}} & 0 & 0 & 0 & \frac{d_{1}^{2}+2 d_{1} d_{2}}{2 E I_{y}} & 0 \\
0 & \frac{2 d_{1}^{3}+3 d_{1}^{2} d_{2}}{6 E I_{x}} & 0 & -\frac{d_{1}^{2}+2 d_{1} d_{2}}{2 E I_{x}} & 0 & 0 \\
0 & 0 & \frac{d_{1}}{E A} & 0 & 0 & 0 \\
\frac{d_{1}^{2}}{2 E I_{y}} & 0 & 0 & 0 & \frac{d_{1}}{E I_{y}} & 0 \\
0 & \frac{d_{1}^{2}}{2 E I_{x}} & 0 & -\frac{d_{1}}{E I_{x}} & 0 & 0 \\
0 & 0 & 0 & 0 & 0 & \frac{d_{1}}{G J}
\end{array}\right] \text {, } \\
& \mathbf{K}_{22}=\left[\begin{array}{cccccc}
\frac{\left(d_{1}+d_{2}\right)^{3}}{3 E I_{y}} & 0 & 0 & 0 & \frac{\left(d_{1}+d_{2}\right)^{2}}{2 E I_{y}} & 0 \\
0 & \frac{\left(d_{1}+d_{2}\right)^{3}}{3 E I_{x}} & 0 & -\frac{\left(d_{1}+d_{2}\right)^{2}}{2 E I_{x}} & 0 & 0 \\
0 & 0 & \frac{d_{1}+d_{2}}{E A} & 0 & 0 & 0 \\
\frac{\left(d_{1}+d_{2}\right)^{2}}{2 E I_{y}} & 0 & 0 & 0 & \frac{d_{1}+d_{2}}{E I_{y}} & 0 \\
0 & \frac{\left(d_{1}+d_{2}\right)^{2}}{2 E I_{x}} & 0 & -\frac{d_{1}+d_{2}}{E I_{x}} & 0 & 0 \\
0 & 0 & 0 & 0 & 0 & \frac{d_{1}+d_{2}}{G J}
\end{array}\right] \text {, } \\
& \overline{\mathbf{K}}_{11}=\mathbf{K}_{11}^{-1}+\mathbf{K}_{11}^{-1} \mathbf{K}_{12}\left(\mathbf{K}_{22}-\mathbf{K}_{21} \mathbf{K}_{11}^{-1} \mathbf{K}_{12}\right)^{-1} \mathbf{K}_{21} \mathbf{K}_{11}^{-1}, \\
& \overline{\mathbf{K}}_{12}=-\mathbf{K}_{11}^{-1} \mathbf{K}_{12}\left(\mathbf{K}_{22}-\mathbf{K}_{21} \mathbf{K}_{11}^{-1} \mathbf{K}_{12}\right)^{-1}, \\
& \overline{\mathbf{K}}_{21}=-\left(\mathbf{K}_{22}-\mathbf{K}_{21} \mathbf{K}_{11}^{-1} \mathbf{K}_{12}\right)^{-1} \mathbf{K}_{21} \mathbf{K}_{11}^{-1}, \\
& \overline{\mathbf{K}}_{22}=\left(\mathbf{K}_{22}-\mathbf{K}_{21} \mathbf{K}_{11}^{-1} \mathbf{K}_{12}\right)^{-1} .
\end{aligned}
$$

\section{Data Availability}

The data used to support the findings of this study are included within the article.

\section{Conflicts of Interest}

The authors declare that there are no conflicts of interest regarding the publication of this paper. 


\section{Authors' Contributions}

Bingyan Li contributed to conceptualization, validation, formal analysis, and writing (original draft). Yuxuan Liu contributed to software and data curation. Rongqiang Liu contributed to resources and funding acquisition. Hongwei Guo contributed to methodology and supervision. Qiang Cong contributed to visualization and project administration. Qiuhong Lin contributed to investigation. Shangling Qiao contributed to writing (review and editing). The second author contributed to quality checking.

\section{Acknowledgments}

This project was supported by the National Natural Science Foundation of China (51835002 and 51675114), the Joint Funds of the National Natural Science Foundation of China (Grant no. U1637207), and the College Discipline Innovation Wisdom Plan in China (Grant no. B07018). These supports are gratefully acknowledged by the authors.

\section{References}

[1] M. Leipold, M. Eiden, C. E. Garner et al., "Solar sail technology development and demonstration," Acta Astronautica, vol. 52, no. 2-6, pp. 317-326, 2003.

[2] A. Lee and J. M. Fernandez, "Mechanics of bistable twoshelled composite booms," in Proceedings of the AIAA Spacecraft Structures Conference, Kissimmee, FL, USA, January 2018 .

[3] J. B. Bai, J. J. Xiong, J. P. Gao, and X. S. Yi, “Analytical solutions for predicting in-plane strain and interlaminar shear stress of ultra-thin-walled lenticular collapsible composite tube in fold deformation," Composite Structures, vol. 97, pp. 64-75, 2013.

[4] Z. Chu and Y. Lei, "Design theory and dynamic analysis of a deployable boom," Mechanism and Machine Theory, vol. 71, pp. 126-141, 2014.

[5] J. A. Firth and M. R. Pankow, "Advanced dual-pull mechanism for deployable spacecraft booms," Journal of Spacecraft and Rockets, vol. 56, no. 2, pp. 569-576, 2019.

[6] Y. Hu, W. Chen, R. Li, and G. Fang, "Mechanical characteristics of deployable composite thin-walled lenticular tubes," Composite Structures, vol. 153, pp. 601-613, 2016.

[7] T. M.. Booms and Trusses, Recent Advances in Gossamer Spacecraft, AIAA, Reston, VA, USA, 2006.

[8] T. Murphey, "A material structural performance index for strain based deloyable trusses," in Proceedings of the 45th AIAA/ASME/ASCE/AHS/ASC Structures, Structural Dynamics And Materials Conference, Palm Springs, CA, USA, June 2004.

[9] M. Eiden, O. Brunner, and C. Stavrinidis, "Deployment analysis of the Olympus Astromast and comparison with test measurements," Journal of Spacecraft and Rockets, vol. 24, no. 1, pp. 63-68, 1987.

[10] G.-Y. Lu, J.-Y. Zhou, G.-P. Cai, G.-Q. Fang, L.-L. Lv, and F.-J. Peng, "Studies of thermal deformation and shape control of a space planar phased array antenna," Aerospace Science and Technology, vol. 93, Article ID 105311, 2019.

[11] C. Gantes, R. Khoury, J. J. Connor, and C. Pouangare, "Modeling, loading, and preliminary design considerations for tall guyed towers," Computers \& Structures, vol. 49, no. 5, pp. 797-805, 1993.
[12] H. Xiao, S. Lu, and X. Ding, "Tension cable distribution of a membrane antenna frame based on stiffness analysis of the equivalent 4-SPS-S parallel mechanism," Mechanism and Machine Theory, vol. 124, pp. 133-149, 2018.

[13] P. M. Páez and B. Sensale, "Analysis of guyed masts by the stability functions based on the Timoshenko beam-column," Engineering Structures, vol. 152, pp. 597-606, 2017.

[14] R. Belevičius, D. Jatulis, D. Rusakevičius, and D. Šešok, "Optimal schemes for tall guyed telecommunication masts," in Proceedings of the 15th International Conference on Civil, Structural and Environmental Engineering Computing, Stirlingshire, UK, 2015.

[15] M. Heydari, H. Moharrami, and H. Yazdani-Paraei, "Nonlinear analysis and optimum design of guyed masts," Journal of Optimization Theory and Applications, vol. 155, no. 3, pp. 1025-1046, 2012.

[16] J. S. Ballaben and M. B. Rosales, "Nonlinear dynamic analysis of a 3D guyed mast," Nonlinear Dynamics, vol. 93, no. 3, pp. 1395-1405, 2018.

[17] S. Alshurafa, H. Alhayek, and D. Polyzois, "Parametric study on the strength and stiffness of FRP meteorological guyed towers," Mechanics of Advanced Materials and Structures, vol. 26, no. 16, pp. 1403-1410, 2019.

[18] R. Belevičius, D. Jatulis, and D. Šešok, "Optimization of tall guyed masts using genetic algorithms," Engineering Structures, vol. 56, pp. 239-245, 2013.

[19] R. Belevičius, D. Jatulis, and D. Šešok, "Some insights on the optimal schemes of tall guyed masts," Journal of Civil Engineering and Management, vol. 19, no. 5, pp. 749-758, 2013.

[20] R. Belevičius, D. Jatulis, D. Rusakevičius, and D. Mačiūnas, "Optimization of rigidly supported guyed masts," Advances in Civil Engineering, vol. 2017, Article ID 4561376, 9 pages, 2017.

[21] V. Elena Parnás, P. Martín Rodríguez, and A. E. Castañeda Hevia, "Structural behavior of guyed mast with asymmetrical anchors," Journal of the Brazilian Society of Mechanical Sciences and Engineering, vol. 35, no. 2, pp. 61-67, 2013.

[22] A. K. Noor, M. S. Anderson, and W. H. Greene, "Continuum models for beam- and platelike lattice structures," AIAA Journal, vol. 16, no. 12, pp. 1219-1228, 1978.

[23] A. K. Noor and W. C. Russell, "Anisotropic continuum models for beamlike lattice trusses," Computer Methods in Applied Mechanics and Engineering, vol. 57, no. 3, pp. 257277, 1986.

[24] A. Salehian, E. M. Cliff, and D. J. Inman, "Continuum modeling of an innovative space-based radar antenna truss," Journal of Aerospace Engineering, vol. 19, no. 4, pp. 227-240, 2006.

[25] D. Zhang, F. Li, F. Shao, and C. Fan, "Evaluation of equivalent bending stiffness by simplified theoretical solution for an FRP-aluminum deck-truss structure," KSCE Journal of Civil Engineering, vol. 23, no. 1, pp. 367-375, 2019.

[26] M. Liu, D. Cao, and D. Zhu, "Equivalent dynamic model of the space antenna truss with initial stress," AIAA Journal, vol. 58, no. 4, pp. 1851-1863, 2020.

[27] J. S. Dai and X. Ding, "Compliance analysis of a three-legged rigidly-connected platform device," Journal of Mechanical Design, vol. 128, no. 4, p. 755, 2006.

[28] Y. Lu and B. Hu, "Analysis of stiffness and elastic deformation for some 3-5-DOF PKMs with SPR or RPS-type legs," Journal of Mechanical Design, vol. 130, no. 10, Article ID 102307, 2008.

[29] F. Hakkak and S. Khoddam, "On calculation of preliminary design parameters for lenticular booms," Proceedings of the Institution of Mechanical Engineers, Part G: Journal of Aerospace Engineering, vol. 221, no. 3, pp. 377-384, 2007. 